\title{
Dynamic Competition between Catch and Slip Bonds in Selectins Bound to Ligands
}

\author{
V. Barsegov ${ }^{*} \dagger$ and D. Thirumalai ${ }^{*, *}$ \\ Department of Chemistry, University of Massachusetts Lowell, Lowell, Massachusetts 01854, Biophysics \\ Program, Institute for Physical Science and Technology, and Department of Chemistry and Biochemistry, \\ University of Maryland, College Park, Maryland 20742
}

Received: August 17, 2006; In Final Form: October 7, 2006

\begin{abstract}
Atomic force measurements of unbinding rates (or off-rates) of ligands bound to a class of cell adhesion molecules from the selectin family show a transition from catch to slip bonds as the value of external force $(f)$ is increased. At low forces $(<10 \mathrm{pN})$, the unbinding rates decrease (catch regime), while, at high forces, the rates increase in accord with the Bell model (slip regime). The energy landscape underlying the catchslip transition can be captured by a two-state model that considers the possibility of redistribution of population from the force-free bound state to the force-stabilized bound state. The excellent agreement between theory and experiments is used to extract the parameters characterizing the energy landscape of the complex by fitting the calculated curves to lifetime data (obtained at constant $f$ ) for the monomeric form of PSGL-1 (sPSGL-1). We used the constant force parameters to predict the distributions of unbinding times and unbinding forces as a function of the loading rate. The general two-state model, which also correctly predicts the absence of catch bonds in the binding of antibodies to selectins, is used to resolve the energy landscape parameters characterizing adhesive interactions of P- and L-selectins with physiological ligands such as sPSGL-1 and endoglycan and antibodies such as G1 and DREG56. Despite high sequence similarity, the underlying shapes of the energy landscape of P-selectin and L-selectin interacting with sPSGL-1 are markedly different. The underlying energy landscape of the selectin cell adhesion complex is sensitive to the nature of the ligand. The unified description of selectins bound to physiological ligands and antibodies in conjunction with experimental data can be used to extract the key parameters that describe the dynamics of cell adhesion complexes.
\end{abstract}

\section{Introduction}

Patrolling of leukocytes during inflammation or tissue injury is mediated by noncovalent $\mathrm{Ca}^{2+}$-dependent interactions between P-, L-, and E-selectin receptors and their specific ligands. Selectins belong to a family of cell adhesion molecules expressed on activated endothelial cells and platelets of blood vessel walls. The receptors bind to specific ligands, such as the counter-receptors ESL-1, podocalyxin, and PSGL-1. ${ }^{1-9}$ These interactions, which facilitate leukocyte tethering (transient capture) and rolling on endothelial cells and platelets, involve a dynamic competition between bond formation under fast loading and rapid bond breakage followed by cell release. Under physiological conditions of blood circulation, the selectin-ligand complex experiences shear stress due to the hydrodynamic force of the flow. It is known that selectins require a critical value of shear to enable adhesion. Below the shear threshold, the lifetime of the tether increases which results in the decrease of rolling velocities. The enhancement of lifetimes of the complex is counterintuitive in light of Bell's suggestion that applied external force might enhance bond rupture rates by lowering the freeenergy barrier between bound and free selectin receptor states. ${ }^{10}$ However, the increase of unbinding rates (also known as offrates) with the wall shear stress is in apparent odds with a shear threshold requirement for selectin-mediated adhesion and rolling, ${ }^{5-7}$ below which fewer cells sustain stable rolling.

* Corresponding authors. (D.T.): Phone: 301-405-4803. Fax: 301-314 9404. E-mail: thirum@glue.umd.edu.; (V.B.): Phone: 978-934-3661. Fax: 978-934-3013. E-mail: Valeri-Barsegov@uml.edu.

University of Massachusetts Lowell.

$\doteqdot$ University of Maryland.
Dembo et al. ${ }^{11}$ hypothesized that shear stress could also prolong bond lifetimes by deforming adhesion complexes into an alternative locked or bound conformation. As shear stress is increased, the locked state could retard bond dissociation at the trailing edge of the cell, thus facilitating formation of the bond at the leading edge and thus stabilizing rolling. These two distinct dynamic responses to external force, namely, an increase in off-rates at high forces and a decrease at lower forces, are referred to as "slip" and "catch" bonds. The dynamics of slip bonds between P-selectins and the physiological ligand PSGL-1 have been extensively studied in flow chamber experiments. ${ }^{3,8,12-15}$ Recently, mechanical unbinding experiments have unambiguously shown that at low forces the lifetime of the bonds can increase which suggests a role for catch bonds under physiological conditions. ${ }^{16,17}$ On the basis of this observation, it has been argued that catch-slip bond dynamics is linked to the shear threshold phenomenon. ${ }^{18}$

Using atomic force microscopy (AFM), Marshall et al. measured the force dependence of the bond lifetimes of P-selectin with monomeric (sPSGL-1) and dimeric (PSGL-1) ligands and with G1, which is a blocking anti-P-selectin monoclonal antibody. By extending the lifetime measurements to the level of forces lower than the level of their fluctuations, ${ }^{16}$ Marshall et al. observed a biphasic pattern of the average bond lifetime $(\langle t\rangle)$ as a function of pulling force $(f)$ for P-selectin adhesion complexes with sPSGL-1 and PSGL-1. ${ }^{16}$ The lifetime $(\langle t\rangle)$ initially increased with $f$, indicating catch bonds. Beyond the critical force $\left(f_{\mathrm{c}}\right)$, corresponding to the longest bond lifetime, $\langle t\rangle=\langle t\rangle_{\max }$, the average bond lifetime decreased with force, as 
expected for slip bonds. ${ }^{16,17}$ The non-monotonic dependence of $\langle t\rangle$ on $f$ depends on the nature of the ligand. For the P-selectinG1 bond, $\langle t\rangle$ decreased exponentially as $f$ increased, which is in accord with the predictions of the Bell model. ${ }^{10}$

The transition from catch to slip bonds observed in many cell adhesion complexes suggests that the energy landscape is complex. The experimental biphasic dynamical response of the P-selectin-PSGL-1 complex is most easily explained using a two-state model, ${ }^{19,20}$ as was convincingly demonstrated by Evans and co-workers ${ }^{19}$ and more generally by Barsegov and Thirumalai (BT). ${ }^{20}$ Such a model has been previously proposed for GTPase Ran complexes, which regulate molecular transport between the cell nucleus and cytoplasm, with the nuclear import receptor importin $\beta 1 .{ }^{21}$ The challenge is not to merely propose a kinetic model to fit the measurements of the dependence of $\langle t\rangle$ on $f$. Apparently, this can be achieved by other kinetic schemes that use rate expressions for forced dissociation that are difficult to justify. ${ }^{22,23,25}$ Some of the models, which build in the catch bond character by artificially postulating negative projection of the force along a dissociation coordinate, ${ }^{2-24}$ have not been applied to selectin bonds to antibodies. While these earlier models have some utility, ${ }^{22-24}$ the more general twostate model is required to describe a wide range of experiments including unbinding of adhesin FimH from a mannose coated surface. ${ }^{26}$ Our goal is to extract the parameters of the energy landscape, such as the location of the transition state and estimates of free-energy barriers using a consistent model for all ligands and various members of the selectin family.

By using two bound states of P-selectins and the Bell model for the dependence of kinetic rates on $f$, we computed the average P-selectin-sPSGL-1 bond lifetime as a function of $f$. Our results are in excellent agreement with experiments. The parameters extracted by fitting the theoretical results to experimental data allowed us to quantitatively map the free-energy landscape of P-selectins complexed with sPSGL-1 and G1 and predict the distributions of unbinding times and unbinding forces. ${ }^{20}$ Using the same formalism, we also showed that, in contrast to P-selectin complexes with physiological ligands, unbinding of P-selectin complexes with the antibody G1 occurs from a single bound state.

The unbinding dynamics of L-selectin complexes with sPSGL-1 and the recently identified PSGL-1-like specific ligand endoglycan also exhibit a similar transition from the catch regime to the slip regime of unbinding. ${ }^{17}$ For example, catch bonds for the L-selectin-sPSGL-1 complex were observed at low forces (below $\approx 60 \mathrm{pN}$ ), coinciding with the shear threshold range $\left(<0.6 \mathrm{dyn} / \mathrm{cm}^{2}\right)$, and slip bonds at higher forces $(>60 \mathrm{pN})$. In contrast, interaction between L-selectin and the antibody DREG56 is characterized only by slip bonds. However, unlike P-selectins, L-selectins were unable to form a double adhesion bond with the dimeric ligand PSGL- $1 ;{ }^{17}$ these findings indicate that catch bonds may be common in specific protein-protein interactions. Both $\mathrm{P}$ - and L-selectin bind to an N-terminal region of PSGL-1 and sialylated and fucosylated $O$-glycan. ${ }^{27-29}$ It is likely that the same binding determinants of P- and L-selectins are responsible for binding of endoglycan. The monoclonal antibodies G1 and DREG56 to the peptide-glycan terminal region block binding of sPSGL-1 and endoglycan to P- and L-selectins.

In the present work, we apply the BT two-state model to analyze the unbinding dynamics of L-selectin complexes with sPSGL-1 and endoglycan and the antibody DREG56. To obtain the free-energy landscape parameters for L-selectin-ligand complexes, theoretical curves of the average bond lifetime are fit to experimental data. ${ }^{17}$ These parameters are used to obtain experimentally testable predictions for the distributions of unbinding times and unbinding forces. The significant changes in the shape of the energy landscape of L- and P-selectins bound to sPSGL-1 underscore the importance of structure specific interactions. The role ligand plays in greatly altering the unbinding free-energy barriers and location of the transition state is underscored by comparing the results for L-selectin in complex with sPSGL-1 and endoglycan. This comparison shows that there is great plasticity in the interaction of L-selectin with ligands.

\section{The Model}

The observation of catch-slip transition in forced unbinding dynamics of selectin-ligand complexes is best explained using a two-state model. ${ }^{19,20}$ In the most general description, the P- or L-selectin receptor $(\mathrm{R})$ adhesion complex with ligand $(\mathrm{L})$ can exist in two distinct bound states, $\mathrm{LR}_{1}$ and $\mathrm{LR}_{2}$ (Figure 1). The transition rates between $\mathrm{LR}_{1}$ and $\mathrm{LR}_{2}$ are $r_{12}=r_{10} \exp \left[-F_{12} /\right.$ $k T]$ and $r_{21}=r_{20} \exp \left[-F_{21} / k T\right]$, where $F_{12}\left(F_{21}\right.$, Figure 1$)$ is the barrier separating $\mathrm{LR}_{1}\left(\mathrm{LR}_{2}\right)$ from $\mathrm{LR}_{2}\left(\mathrm{LR}_{2}\right)$. In the Kramer type theory, the prefactors $r_{10}$ and $r_{20}$ depend on the shapes of the basins corresponding to $\mathrm{LR}_{1}$ and $\mathrm{LR}_{2}$ and the regions near the transition states. Conformational transitions obey the detailed balance condition

$$
K_{\text {eq }} \equiv \frac{r_{12}}{r_{21}}=\frac{r_{10}}{r_{20}} \mathrm{e}^{-\Delta F / k T}
$$

where $K_{\text {eq }}$ is the equilibrium constant and $\Delta F$ is the free energy of stability of $\mathrm{LR}_{1}$ with respect to $\mathrm{LR}_{2}$ (Figure 1 ).

Upon application of a stretching force, $f, \Delta F$ is modulated by an amount, $\sigma f$, where $\sigma=x_{1}-x_{2}$, the conformational compliance, is the difference in the distance between energy minima of states $\mathrm{LR}_{1}$ and $\mathrm{LR}_{2}$ and the transition state. Force shifts the detailed balance by $\exp [\sigma f / k T]$, so that

$$
K_{\mathrm{eq}} \rightarrow K_{\mathrm{eq}}(f)=K_{\mathrm{eq}} \mathrm{e}^{\sigma f / k T}
$$

which results in a new equilibrium with the force-dependent constant $K_{\text {eq }}(f)$. In the presence of force, the free-energy landscape for the P-selectin-ligand unbinding is also altered. The bond rupture rates $\left(k_{1}(f)\right.$ and $\left.k_{2}(f)\right)$ are given by ${ }^{10}$

$$
k_{1}=k_{10} \mathrm{e}^{y_{1} f / k T} \quad \text { and } \quad k_{2}=k_{20} \mathrm{e}^{y_{2} f / k T}
$$

where $k_{10}$ and $k_{20}$ are the force-free bond-breakage rates and $y_{1}$ and $y_{2}$ are the minimal adhesion bond lengths at which the complex becomes unstable (distances between energy minima of $\mathrm{LR}_{1}$ and $\mathrm{LR}_{2}$ and their respective transition states). Although the Bell model is only approximate, ${ }^{30}$ it well describes the dissociation of single selectin bonds (in the slip regime) over a broad range of loading rates. ${ }^{31} \mathrm{We}$ assume that, in the presence of $f$, rebinding of $\mathrm{P}$ - and $\mathrm{L}$-selectin to the ligand is negligible.

The unbinding kinetics for the two-state model (Figure 1) is complicated because of a number of competing rate processes. To fit the experimental data for $\langle t\rangle$ as a function of $f$, we required only four parameters $\left(r_{12}, r_{21}, k_{1}\right.$, and $\left.k_{2}\right)$. In microscopic terms, the complete characterization of the free-energy landscape (Figure 1) is given by the parameters $r_{10}, r_{20}, x_{1}, x_{2}, k_{10}, k_{20}, y_{1}$, and $y_{2}$. The number of parameters may be reduced by assuming that $\mathrm{LR}_{1}$ and $\mathrm{LR}_{2}$ are in equilibrium. While such an assumption is experimentally satisfied for unbinding of sPSGL-1 from P-selectin, ${ }^{19}$ we have chosen to treat the kinetic equations in 


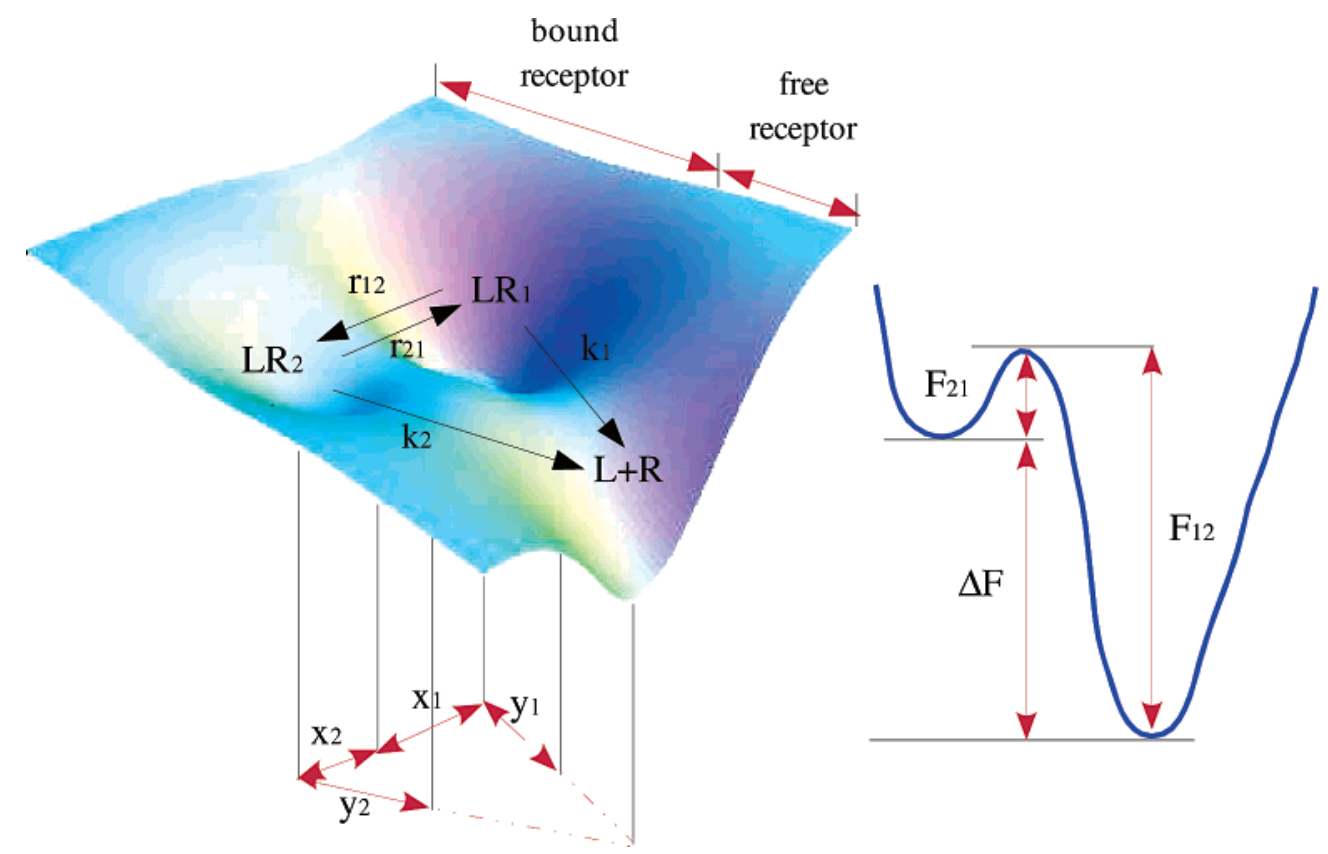

Figure 1. Schematic of the energy landscape for complexes involving cell adhesion molecules (left) with parameters characterizing unbinding dynamics. The 1D profile on the right shows the conformational free energy. Upon application of force, the force-free equilibrium shifts, resulting in redistribution of the population of bound states $\mathrm{LR}_{1}$ and $\mathrm{LR}_{2}$. Force-induced alteration of the free-energy landscape is dynamically coupled to forced unbinding.

the most general terms. The multiple unbinding pathways that lead to the catch-slip transitions can be seen by the modulation in the force-induced population of $\mathrm{LR}_{1}$ and $\mathrm{LR}_{2}$ and the rupture rates $k_{1}$ and $k_{2}$. The force-induced conformational transitions are determined by $r_{12}(f)$ and $r_{21}(f)$ or equivalently by $K_{\text {eq }}(f)$ and $\sigma$ (eq 2). When $f$ is applied to a selectin complex with a ligand, there is a shift in the population from

$$
P_{1}(0) \equiv 1 /\left(K_{\mathrm{eq}}+1\right) \quad \text { and } \quad P_{2}(0) \equiv K_{\mathrm{eq}} /\left(K_{\mathrm{eq}}+1\right)
$$

to the new equilibrium with populations

$$
P_{1}(f) \equiv 1 /\left(K_{\mathrm{eq}}(f)+1\right) \quad \text { and } \quad P_{2}(f) \equiv K_{\mathrm{eq}}(f) /\left(K_{\mathrm{eq}}(f)+1\right)
$$

The population shift occurs on a time scale of $\tau_{\mathrm{c}}(f)=\left(r_{12}(f)\right.$ $\left.+r_{21}(f)\right)^{-1}$. Force alters both the rates $k_{1}$ and $k_{2}$ of unbinding and the rates $r_{12}$ and $r_{21}$ of conformational transitions. As a result, the time scale in which population shift occurs competes with the unbinding time scale, $\tau_{1}(f)=k_{1}(f)^{-1}$ (corresponding to bond rupture from $\mathrm{LR}_{1}$ ) and $\tau_{2}(f)=k_{2}(f)^{-1}$ (representing unbinding from $\mathrm{LR}_{2}$ ). Thus, $\tau_{\mathrm{c}}, \tau_{1}$, and $\tau_{2}$ reflect intrinsic structural and dynamic properties of the cell adhesion complex in question and completely determine the unbinding dynamics. The relevance of catch and slip bonds depends on the competition between $\tau_{\mathrm{c}}, \tau_{1}$, and $\tau_{2}$. If $\tau_{\mathrm{c}} / \tau_{1} \ll 1$, then $\mathrm{LR}_{1}$ and $\mathrm{LR}_{2}$ are in fast equilibrium, as might be the case for the P-selectinsPSGL-1 complex. If the value of $f$ is such that $\tau_{1}(f) / \tau_{2}(f) \sim$ $O(1)$, then rupture of the complex occurs nearly simultaneously from $\mathrm{LR}_{1}$ and $\mathrm{LR}_{2}$. This is likely to take place in the transition force $\left(f^{*}\right)$ between catch and slip bonds that is given by $f^{*} \approx$ $k_{\mathrm{B}} T \log \left[K_{\mathrm{eq}} / \sigma\right]$.

\section{Distributions of Bond Lifetimes at Constant Force}

When $f$ is constant, the populations $P_{1}(t)$ and $P_{2}(t)$ of states $\mathrm{LR}_{1}$ and $\mathrm{LR}_{2}$ can be calculated by solving the system of coupled ordinary differential equations

$$
\begin{aligned}
& \frac{\mathrm{d} P_{1}}{\mathrm{~d} t}=-\left(r_{12}+k_{1}\right) P_{1}+r_{21} P_{2} \\
& \frac{\mathrm{d} P_{2}}{\mathrm{~d} t}=r_{12} P_{1}-\left(r_{21}+k_{2}\right) P_{2}
\end{aligned}
$$

subject to the initial conditions given in eq 4 . By converting eq 6 into algebraic equations using the Laplace transform, the populations $\bar{P}_{1}(z)$ and $\bar{P}_{2}(z)$ of the P-selectin bound states $\mathrm{LR}_{1}$ and $\mathrm{LR}_{2}$ in the Laplace domain, defined by

$$
\bar{f}(z)=\int_{0}^{\infty} \mathrm{d} t \mathrm{e}^{-z t} f(t)
$$

can be computed using

$$
\bar{P}_{i}(z)=\sum_{j=1,2} \bar{G}_{i j}(z) \bar{P}_{j}(0)
$$

where $\bar{P}_{1}(0)=P_{1}$ and $\bar{P}_{1}(0)=P_{2}$ are given by eq 4 . The matrix elements of the Green function $\left(\bar{G}_{i j}(z)\right), i, j=1,2$, are

$$
\begin{aligned}
& \bar{G}_{11}(z)=\frac{\left(z+k_{2}+r_{21}\right)}{\operatorname{det}}, \quad \bar{G}_{22}(z)=\frac{\left(z+k_{1}+r_{12}\right)}{\operatorname{det}}, \\
& \bar{G}_{12}(z)=\frac{r_{21}}{\operatorname{det}}, \quad \bar{G}_{21}(z)=\frac{r_{12}}{\operatorname{det}}
\end{aligned}
$$

where det $=\left(z+k_{1}\right)\left(z+k_{2}\right)+r_{21}\left(z+k_{1}\right)+r_{12}\left(z+k_{2}\right)$. The populations in real time $\left(P_{1,2}(t)\right)$ are obtained using $P_{1,2}(t)=$ $L^{-1}\left[\bar{P}_{1,2}(z)\right]$, where $L^{-1}$ denotes the inverse Laplace $(z \rightarrow t)$ transform. The solution to eq 6 is

$$
\begin{aligned}
& P_{1}(t)=\frac{P_{1}(0)\left(k_{2}+z_{1}\right)+r_{21}}{z_{1}-z_{2}} \mathrm{e}^{z_{1} t}-\frac{P_{1}(0)\left(k_{2}+z_{2}\right)+r_{21}}{z_{1}-z_{2}} \mathrm{e}^{z_{2} t} \\
& P_{2}(t)=\frac{P_{2}(0)\left(k_{1}+z_{1}\right)+r_{12}}{z_{1}-z_{2}} \mathrm{e}^{z_{1} t}-\frac{P_{2}(0)\left(k_{1}+z_{2}\right)+r_{12}}{z_{1}-z_{2}} \mathrm{e}^{z_{2} t}
\end{aligned}
$$


where $z_{1,2}=-\left[k_{1}+k_{2}+r_{12}+r_{21} \pm \sqrt{D}\right] / 2$ are the eigenvalues, which determine the effective unbinding time scales, $1 / z_{\alpha}(\alpha=1,2)$, and $D=\left(k_{1}+k_{2}+r_{12}+r_{21}\right)^{2}-4\left(k_{1} k_{2}\right.$ $\left.+k_{1} r_{21}+k_{2} r_{12}\right)$. The ensemble average distribution of lifetimes $(P(t))$ is given by the sum of contribution from states $\mathrm{LR}_{1}$ and $\mathrm{LR}_{2}$ :

$$
P(t)=P_{1}(t)+P_{2}(t)
$$

and the ensemble average bond lifetime is

$$
\langle t\rangle=\int_{0}^{\infty} \mathrm{d} t P(t) t
$$

In the limit of slow conformational fluctuations between the two bound states, that is, when $r_{12}, r_{21} \ll k_{1}, k_{2}$, eigenvalues reduce to $z_{\alpha} \approx-k_{\alpha}(\alpha=1,2)$. In this limit, the rupture from the states $\mathrm{LR}_{1} \rightarrow \mathrm{L}+\mathrm{R}$ and $\mathrm{LR}_{2} \rightarrow \mathrm{L}+\mathrm{R}$ is decoupled. As a result,

$$
P(t, f) \approx P_{1}(0) \mathrm{e}^{-k_{1}(f) t}+P_{2}(0) \mathrm{e}^{-k_{2}(f) t}
$$

In the opposite extreme, $r_{12}, r_{21} \gg k_{1}, k_{2}, z_{1} \ll z_{2}$, and, because the decay channels $\mathrm{LR}_{1} \rightarrow \mathrm{L}+\mathrm{R}$ and $\mathrm{LR}_{2} \rightarrow \mathrm{L}+\mathrm{R}$ are coupled, unbinding dynamics is determined by the total unbinding rate, $k_{1}+k_{2}$,

$$
P(t, f) \approx \mathrm{e}^{-\left(k_{1}(f)+k_{2}(f)\right) t}
$$

The fast population redistribution case $\left(r_{12}, r_{21} \gg k_{1}, k_{2}\right)$ was previously used by Evans et al. to analyze the experimental data. ${ }^{19}$ Such a single-exponential decay was used by others to fit the dependence of $\langle t\rangle$ on $f$. The meaning of $k_{1}(f)$ and $k_{2}(f)$ in the present model and the one in ref 19 are completely different.

Using eqs 11 and 12, we calculated the distribution of lifetimes $(P(t, f))$ as a function of $f$ and the average lifetime $(\langle t(f)\rangle)$ versus $f$ characteristics for specific complexes: P-selectinsPSGL-1, L-selectin-sPSGL-1, and L-selectin-endoglycan (Figure 2). We used the same model to calculate $P(t, f)$ for complexes of P- and L-selectins with the antibodies G1 and DREG56, respectively (Figure 3). The model parameters of the energy landscape for P-selectin complexes with sPSGL-1 and G1 were obtained by fitting the theoretical curves of $\langle t(f)\rangle$ versus $f$ to the experimental data of Marshall et al. ${ }^{16}$ We estimated the corresponding energy landscape parameters for L-selectin complexes with sPSGL-1, endoglycan, and DREG56 by fitting the theoretically computed $\langle t(f)\rangle$ values to the experiments of Sarangapani et al. ${ }^{17}$ (all calculations are done at room temperature). The agreement between theory and experiments is excellent. We list the energy landscape parameters for the various complexes in Table 1 . In what follows, we discuss the results in detail.

P-Selectin. Let us first analyze the unbinding dynamics of P-selectins bound to ligands (top panels in Figures 2 and 3). By comparing $\langle t\rangle$ for P-selectin complexes with sPSGL-1 and G1, we observe a qualitative difference in the force profiles of $\langle t\rangle$ for these ligands. For the complex with sPSGL-1, $\langle t\rangle$ exhibits a sharp growth at low $f$, followed by a crossover to a precipitous decay to zero at higher force, marking the transition from the catch regime to the slip regime of unbinding. In contrast, $\langle t\rangle$ for G1 starts off at $\langle t\rangle \approx 5 \mathrm{~s}$ (not shown) and decays to zero at higher force. Since $K_{\mathrm{eq}}=0.13$ in the absence of force for the P-selectin-sPSGL-1 complex, binding of P-selectins with sPSGL-1 stabilizes the $\mathrm{LR}_{1}$ state of the P-selectin.

For the antibody G1, $K_{\text {eq }}=1\left(r_{20}=r_{10}=10.2 \mathrm{~s}^{-1}\right)$, indicating that both states $\mathrm{LR}_{1}$ and $\mathrm{LR}_{2}$ are equally stable, and thus, there
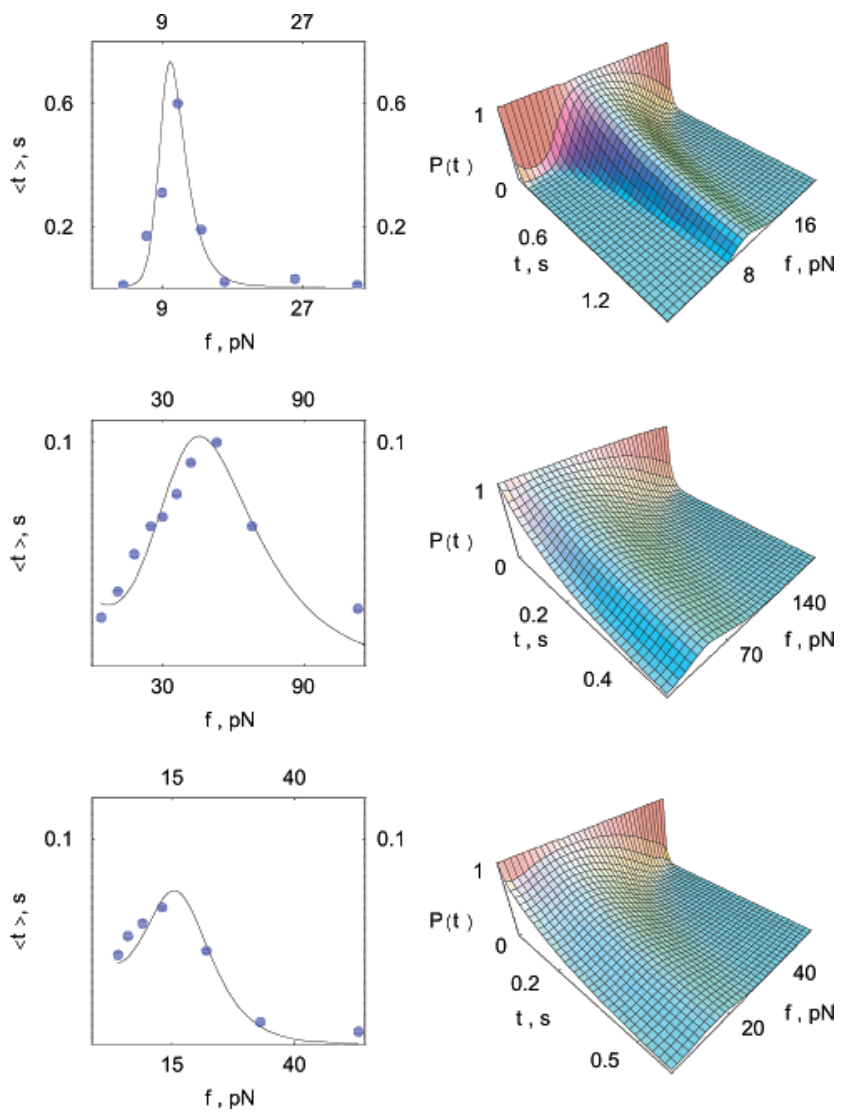

Figure 2. Average bond lifetime $(\langle t(f)\rangle)$ (left panels) and the population of bound states $(P(t, f))$ (right panels) vs pulling force $(f)$ for the P-selectin complex with sPSGL-1 (top) and the L-selectin complex with sPSGL-1 (middle) and endoglycan (bottom). The blue circles are experimental data points from Figure 3 in ref 16 and Figure 4 in ref 17. The initial growth of $\langle t\rangle$ for $f<f_{\mathrm{c}}\left(f_{\mathrm{c}} \approx 10,50\right.$, and $15 \mathrm{pN}$ for the complexes P-selectin-sPSGL-1, L-selectin-sPSGL-1, and L-selectinendoglycan, respectively) that is followed by decay to zero for $f>f_{\mathrm{c}}$ marks the transition from the catch regime to the slip regime. Note the redistribution of $P(f, t)$ at longer unbinding times as $f$ is increased to $f_{\mathrm{c}}$, followed by narrowing at shorter times for $f>f_{\mathrm{c}}$.

is effectively only one bound state. In this case, $k_{10}=k_{20}=$ $0.35 \mathrm{~s}^{-1}$ and $y_{1}=y_{2}=0.32 \mathrm{~nm}$, leading to a landscape with one minimum. In other words, the two bound states are indistinguishable. P-Selectins form a stronger adhesion complex with G1 compared to sPSGL-1; $k_{10}=k_{20}$ for G1 is smaller than $k_{10}$ for sPSGL- 1 , and $y_{1}=y_{2}$ is smaller than $y_{1}$ or $y_{2}$ for sPSGL-1. These findings imply that complexes of P-selectin with $\mathrm{G} 1$ are less sensitive to the applied force. By comparing the values of $y_{1}$ and $y_{2}$ for P-selectin interacting with SPSGL-1 and G1, we conclude that the P-selectin-sPSGL-1 complex is plastic, whereas P-selectin-G1 is brittle ( $y_{1}=y_{2}$ is short). These ideas, which are well-known in material science, have recently been used to discuss the response of biomolecules subject to tension. ${ }^{24,32}$

The role played by the pulling force is twofold: it facilitates unbinding from state $\mathrm{LR}_{1}$ and funnels the population of the P-selectin-sPSGL-1 complex into the locked state $\mathrm{LR}_{2}$. As a result, stretching of complexes with sPSGL-1 couples conformational relaxation and unbinding in the entire range of the applied pulling force. Below $f \approx 3 \mathrm{pN}, r_{12} \approx r_{10}, r_{21} \approx r_{20}, k_{1}$ $\approx k_{10}$, and $k_{2} \approx k_{20}$, and since $P_{1}(0, f)>P_{2}(0, f)$, unbinding occurs from predominantly the $\mathrm{LR}_{1}$ state. However, even at intermediate values of force, $3 \mathrm{pN}<f \leq 12 \mathrm{pN}, k_{1} \gg k_{2}, r_{12} \gg r_{21}$, and since $k_{1} \ll r_{12}$, due to $y_{1} \ll x_{1}, x_{2}, P_{2}(0, f)>P_{1}(0, f)$. In this force regime, sPSGL-1 unbinding dynamics is dominated by 

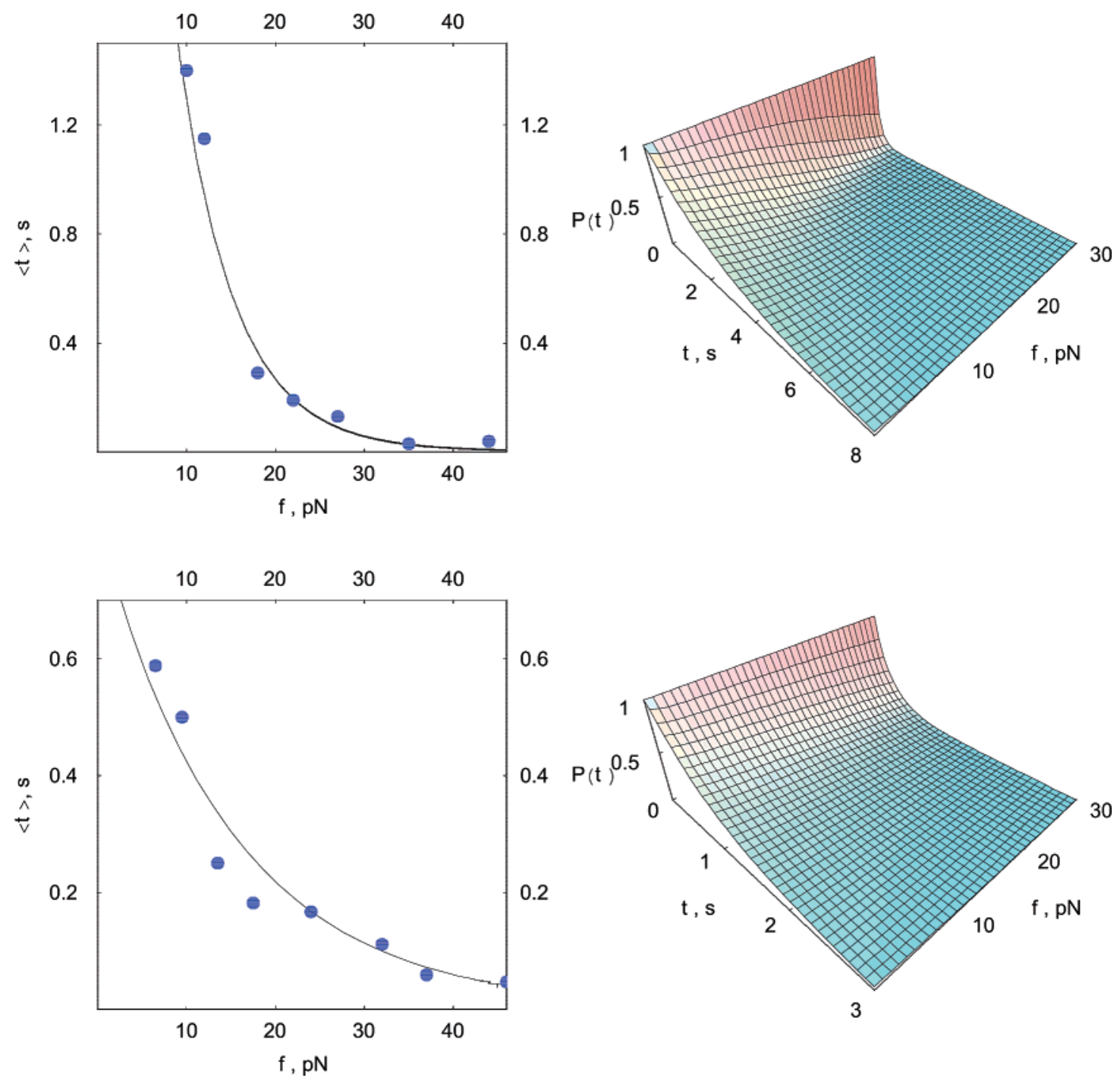

Figure 3. Average bond lifetime $(\langle t(f)\rangle)$ (left panels) and the population of bound states $(P(t, f))$ (right panels) vs pulling force $(f)$ for the P-selectin complex with G1 (top) and the L-selectin-DREG56 complex (bottom). The blue circles are experimental data points from Figure 3 in ref 16 and Figure 4 in ref 17. $\langle t(f)\rangle$ decays with $f$, and $P(t)$ narrows at shorter $\langle t\rangle$ as $f$ is increased.

TABLE 1: Calculated Energy Landscape Parameters (Figure 1) for Specific Ligands sPSGL-1 and Endoglycan and Antibody G1 and DREG56 Unbinding Kinetics from P- and L-Selectins (The Parameters Are Obtained by Fitting the Measured ${ }^{16}$ Average Bond Lifetime $(\langle t\rangle)$ vs $f$ for P-Selectin Complexes with sPSGL-1 and G1 and L-Selectin Complexes with sPSGL-1, Endoglycan, and DREG56 ${ }^{17}$ to the Theoretical Results Based on the Two-State Model)

\begin{tabular}{|c|c|c|c|c|c|c|c|c|}
\hline complex & $\begin{array}{c}r_{10} \\
(1 / \mathrm{s})\end{array}$ & $\begin{array}{c}r_{20} \\
(1 / \mathrm{s})\end{array}$ & $\begin{array}{c}x_{1} \\
(\mathrm{~nm})\end{array}$ & $\begin{array}{c}x_{2} \\
(\mathrm{~nm})\end{array}$ & $\begin{array}{c}k_{10} \\
(1 / \mathrm{s})\end{array}$ & $\begin{array}{c}k_{20} \\
(1 / \mathrm{s})\end{array}$ & $\begin{array}{c}y_{1} \\
(\mathrm{~nm})\end{array}$ & $\begin{array}{c}y_{2} \\
(\mathrm{~nm})\end{array}$ \\
\hline P-selectin-sPSGL-1 & 5.1 & 40.2 & 5.1 & 0.5 & 100.1 & 0.05 & 1.5 & 1.1 \\
\hline L-selectin-sPSGL-1 & 7 & 48 & 0.3 & 0.74 & 0.9 & 105 & 0.1 & 0.12 \\
\hline L-selectin-endoglycan & 5 & 10 & 0.3 & 3.1 & 0.55 & 35 & 0.4 & 2.1 \\
\hline $\mathrm{P}$-selectin-G1 & 10.2 & 10.2 & 3.05 & 3.05 & 0.35 & 0.35 & 0.32 & 0.32 \\
\hline L-selectin-DREG56 & 30.2 & 30.2 & 2.9 & 2.9 & 1.1 & 1.1 & 0.14 & 0.14 \\
\hline
\end{tabular}

smallest eigenvalue, $z_{1}$, corresponding to the longest time scale, $1 / z_{1}$, which becomes $z_{1} \approx-\left(r_{12}-\sqrt{D}\right) / 2$, where $D \approx r_{12}^{2}-$ $4 k_{1} r_{21}-4 k_{2} r_{12}$. It is easy to show, by expanding $\sqrt{D}$ in powers of $\left(k_{1} r_{21}+k_{2} r_{12}\right) / r_{12}{ }^{2}$ and retaining only terms to the first order, that the distribution of bond lifetimes is determined by the effective unbinding rate

$$
k_{\mathrm{eff}}=k_{1} / K_{\mathrm{eq}}(f)+k_{2}
$$

Because $K_{\text {eq }} \ll 1$ and $k_{1} \gg k_{2}$ at low forces, $k_{\text {eff }}$ is largely dominated by the first term in eq 15 and is given by the catch rate constant
The effective rate $\left(k_{\text {eff }}\right)$ decreases with $f$ exponentially due to the increase in $K_{\text {eq }}(f)$ (eqs 1 and 2). Because $k_{1} / K_{\text {eq }}(f) \ll k_{2}$, unbinding at $f$ greater than a critical force of $f_{\mathrm{c}} \approx 10 \mathrm{pN}$ is dominated by the decay from state $\mathrm{LR}_{2}$ with the slip rate constant

$$
k_{\text {eff }}=k_{\text {slip }} \approx k_{2}
$$

which increases with $f$ exponentially. The resulting dual behavior of the average bond lifetime $(\langle t\rangle)$, which exhibits sharp growth at low $f$ reaching a maximum at $\left(f_{\mathrm{c}},\langle t\rangle_{\max }\right) \approx(10 \mathrm{pN}, 0.7 \mathrm{~s})$, 
and decays to zero for $f>f_{\mathrm{c}}$ manifests the transition from catch to slip bonds (Figure 2). In contrast, $\langle t\rangle$ for antibody G1 is a decaying function in the entire range of $f$.

The large differences in the nature of unbinding of sPSGL-1 and G1 from P-selectin is better visualized by comparing the 2D surface of the population of bound states for the P-selectin complex with sPSGL-1 (Figure 2) with the same quantity for P-selectin-G1 (Figure 3). Due to force-induced population redistribution between $\mathrm{LR}_{1}$ and $\mathrm{LR}_{2}$, increase of $f$ to $10 \mathrm{pN}$ for sPSGL-1 results in the redistribution of $P(t)$ around longer lifetimes, which corresponds to the acsending part of the curve for $\langle t\rangle$ (catch regime). In this force range, redistribution of the initial (force-free) population of bound states $\left(P_{1}(0) \approx 0.9, P_{2}(0)\right.$ $\approx 0.1)$ into a force-dependent population $\left(P_{1}(f) \approx P_{2}(f)\right)$ competes with unbinding. When $f$ exceeds $10 \mathrm{pN}$, the dynamics of unbinding is determined by the bond rupture from the more populated state $\mathrm{LR}_{2}\left(P_{2}(f) \approx 1\right)$. As a result, $P(t)$ narrows toward shorter lifetimes, resulting in the decay of $\langle t\rangle$ (slip regime). In contrast, due to the equality of $r_{12}$ and $r_{21}\left(r_{10}=r_{20}, x_{1}=x_{2}\right)$ for P-selectin complexes with $\mathrm{G} 1$, the growth of $k_{1}=k_{2}$ with $f$ favors increasingly shorter lifetimes, which results only in the observation of slip bonds for $G 1$ over the entire range of $f$.

L-Selectin. Similar to P-selectin-sPSGL-1 complexes, constant force-induced unbinding of sPSGL-1 and endoglycan from L-selectins is characterized by the transition from catch bonds that dominate at low forces to slip bonds observed at higher forces (Figure 2). However, this crossover from catch to slip behavior occurs at a higher force compared to the case of P-selectins, namely, at $f_{\mathrm{c}} \approx 40 \mathrm{pN}$ for sPSGL- 1 and $f_{\mathrm{c}} \approx 15 \mathrm{pN}$ for endoglycan. More importantly, the catch-slip transition in L-selectin complexes is not as sharp as that in the P-selectinsPSGL-1 complex. Comparison of the two-dimensional distribution function $P(t, f)$ (Figure 2) shows dramatically that the responses of $\mathrm{P}$-selectin and L-selectin complexes to pulling force are very different. In addition to the higher value of $f_{c}$, the width of the transition in the L-selectin complexes is considerably greater than that in P-selectin bound to sPSGL-1. This could mean that there are much larger fluctuations in L-selectin bound to ligands. The shorter average lifetime $(\approx 0.1 \mathrm{~s}$ for sPSGL- 1 and $0.07 \mathrm{~s}$ for endoglycan) compared to the P-selectin-sPSGL-1 complex $(\approx 0.7 \mathrm{~s})$ also implies that complexes of L-selectins with physiological ligands are less stable compared with complexes involving P-selectins. Just as in P-selectin-G1 complexes, the L-selectin complex with the antibody DREG56 exhibits only slip bonds (Figure 3). However, unlike stronger complexes of G1 compared to sPSGL- 1 in the range $0 \mathrm{pN} \leq f$ $\leq 40 \mathrm{pN}$, the L-selectin-DREG56 complex is more stable in the $0-40 \mathrm{pN}$ range and less stable at $f \geq 40 \mathrm{pN}$ compared to the complex with sPSGL-1 but more stable than the complex with endoglycan (Figure 3).

The force-free bound state $\mathrm{LR}_{1}$ for the sPSGL-1 complex with L-selectin is less stable $\left(K_{\text {eq }}=0.15\right)$ compared to $\mathrm{LR}_{1}$ for P-selectin $\left(K_{\mathrm{eq}}=0.13\right)$ and, thus, is less populated at zero force (compare, e.g., $P_{1}(0) \approx 0.9$ for P-selectins with $P_{1}(0) \approx 0.85$ for L-selectins). For complexes of L-selectins with endoglycan, both states $\mathrm{LR}_{1}$ and $\mathrm{LR}_{2}$ are almost equally stable $\left(K_{\mathrm{eq}}=0.5\right)$ in the absence of force, implying that $P_{1}(0) \approx 0.7$ and $P_{2}(0) \approx$ 0.3 . The reduced stability preferred at the zero force bound state $L_{1}$ relative to the force-stabilized state $L_{2}$ is in part responsible for the reduced strength and lower amplitude of $\langle t\rangle$ for complexes involving L-selectins. Similar to complexes of P-selectins with antibody $\mathrm{G} 1, K_{\mathrm{eq}}=1, x_{1}=x_{2}, k_{10}=k_{20}$, and $y_{1}=y_{2}$ for L-selectin-DREG56 complexes, leading to $a$ landscape with one minimum. Unlike P-selectin-sPSGL-1 complexes for which $x_{1} \gg x_{2}$, the same ligand complexed with L-selectins is characterized by $x_{1} \sim x_{2}$. The distance between $\mathrm{LR}_{1}$ and $\mathrm{LR}_{2}$ for the sPSGL-1 complex with P-selectin $(5.6 \mathrm{~nm})$ is 5 times longer compared with the same distance for L-selectin $(\approx 1 \mathrm{~nm})$, and the distances from bound states to their transition states $(1.5$ and $1.1 \mathrm{~nm})$ are an order of magnitude longer for P-selectins compared with the same distances for L-selectins $\left(y_{1} \approx y_{2} \approx 0.1 \mathrm{~nm}\right)$. This implies that bound states of P-selectins are structurally more distinct compared to states $L_{1}$ and $L_{2}$ of L-selectins. In addition, the binding interface of L-selectins is much stiffer compared to the P-selectin interface which might explain the inability of L-selectins to form a double bond with the dimeric ligand PSGL-1. ${ }^{17}$ Similar to unbinding of sPSGL-1 from P-selectins, due to $k_{20} \gg k_{10}$ and $y_{2}>y_{1}$ (see Table 1), unbinding is dominated by the decay from one state. However, this state is the force-stabilized state $\mathrm{LR}_{2}$ for L-selectins, whereas the high flux in the dissociation process (in the low force regime) occurs through the $\mathrm{LR}_{1}$ state $\left(k_{10} \gg k_{20}\right)$ for P-selectins. The quantitative analysis using the two-state model also shows that L-selectin complexes exhibit only a weak signature of the catch-slip transition.

By comparing the unbinding parameters for endoglycan and sPSGL-1 bound to L-selectin, we see that, aside from the difference in force-free population, $P_{1}(0) \approx P_{2}(0)$ (for endoglycan) versus $P_{1}(0) \gg P_{2}(0)$ (for sPSGL-1), these ligands show similar unbinding patterns. Similar to the L-selectin-sPSGL1 complex, $x_{1}<x_{2}, k_{10} \ll k_{20}$, and $y_{1}<y_{2}$ for the L-selectin complex with endoglycan. However, bound states are structurally distinct for endoglycan (compare, e.g., $x_{2}$ for endoglycan with $x_{1} \sim x_{2}$ for sPSGL-1), with $\mathrm{LR}_{1}$ being very close to the transition state $\left(x_{1}=0.3 \mathrm{~nm}\right)$ and more distant from the unbound state $\left(y_{1}=0.4 \mathrm{~nm}, y_{2}=2.1 \mathrm{~nm}\right)$ compared to complexes with sPSGL-1 $\left(y_{1}=0.1 \mathrm{~nm}, y_{2}=0.12 \mathrm{~nm}\right)$. Due to the longer values of $y_{1}$ and $y_{2}$ for endoglycan, $\langle t\rangle$ shows a faster decay with $f$ compared with $\langle t\rangle$ for sPSGL-1 (Figure 2). P- and L-selectin complexes with antibodies are characterized by more stable bonds.

Unbinding of P- and L-selectins with antibodies G1 and DREG56 is characterized only by slip bonds (Figure 3 ). The equality of the model parameters for bound states for $\mathrm{G} 1$ and DREG56 (Table 1) indicates that unbinding of antibodies involves a single bound state $L R$ which leads to the free-energy landscape with one minimum. Because $k_{10}=k_{20}=1.1 \mathrm{~s}^{-1}$ for the L-selectin-DREG56 complex exceeds $k_{10}=k_{20} \approx 0.35 \mathrm{~s}^{-1}$ for the P-selectin complex with G1, the average lifetime for the L-selectin-DREG56 bond is shorter than $\langle t\rangle$ for the P-selectin-G1 bond at low $0 \mathrm{pN} \leq f \leq 25 \mathrm{pN}$ forces. However, because $y_{1}=y_{2}=0.14 \mathrm{~nm}$ for DREG56 is shorter than $y_{1}=$ $y_{2}=0.32 \mathrm{~nm}$ for G1, $\langle t\rangle$ for the L-selectin-DREG56 complex shows a slower decay with $f$, compared to $\langle t\rangle$ for the P-selectinG1 complex, and results in longer lifetimes at higher $f>25 \mathrm{pN}$ forces (Figure 3).

\section{Distributions of Unbinding Times and Unbinding Forces: Loading Rate Dependence}

The excellent agreement between model and experiment validates the two-state description. By fixing the energy landscape parameters (Table 1), obtained under constant force, we computed the distributions of unbinding times $(p(t))$ and unbinding forces $(p(f))$ when the loading rate is varied. When the pulling force is increased at the loading rate $\left(r_{\mathrm{f}}\right)$, the rate constants $k_{1}, k_{2}, r_{12}$, and $r_{21}$ become time-dependent. The populations $P_{1}(t)$ and $P_{2}(t)$ are computed numerically by solving eq 6 . The total population of bound states is given by 


$$
\langle\dot{P}(t)\rangle=-k_{1}(t) P_{1}(t)-k_{2}(t) P_{2}(t)
$$

and $p(t)$ is obtained from $P(t)$ using

$$
p(t)=k_{1}(t) P_{1}(t)+k_{2}(t) P_{2}(t)
$$

From $p(t)$, we compute $p(f)$ using the transformation

$$
(t, p(t)) \rightarrow\left(r_{\mathrm{f}} t, p(f)\right)
$$

where $p(f)=\left(1 / r_{\mathrm{f}}\right)\left[k_{1}(f) P_{1}\left(f / r_{\mathrm{f}}\right)+k_{2}(f) P_{2}\left(f / r_{\mathrm{f}}\right)\right]$. The most probable rupture force $\left(f^{*}\right)$ is obtained by finding a maximum of $p(f)$, that is, $[\mathrm{d} p(f) / \mathrm{d} t]_{f=f^{*}}=0$.

The distribution functions $p(f)$ and $p(t)$ for complexes of $\mathrm{P}$ and L-selectins with sPSGL-1 and L-selectins with endoglycan are displayed in Figure 4. For all three complexes, $p(f)$ decreases from its initial amplitude but develops a peak at a loading rate of $r_{\mathrm{f}}>300 \mathrm{pN} / \mathrm{s}$ and shifts toward higher forces at faster $r_{\mathrm{f}}$. The width of $p(f)$ does not vary appreciably with $r_{\mathrm{f}}$. The values of $y$ for the P-selectin-sPSGL-1 complex exceed by an order of magnitude those for complexes involving L-selectins, while $k_{10}$ and $k_{20}$ are roughly equal for all three complexes. As a result, $p(f)$ for P-selectin complexes with sPSGL-1 decays to zero at a lower $<10 \mathrm{pN}$ force compared to $p(f)$ for complexes of L-selectin at similar loading rates (compare, e.g., $p(f)$ for $r_{\mathrm{f}}>$ $1000 \mathrm{pN} / \mathrm{s}$ ). This implies that adhesive interactions involving P-selectins are weaker compared with those involving Lselectins. Although both bound states contribute to unbinding, the P-selectin-sPSGL-1 complex is characterized by $y_{2} \approx y_{1}$, and hence, $p(t)$ has only one single peak at faster loading rates. In contrast, because $y_{2} \gg y_{1}$ for L-selectin complexes with sPSGL-1 and endoglycan, $p(f)$ for L-selectin-sPSGL-1 has an additional peak already at $r_{\mathrm{f}} \approx 2000 \mathrm{pN} / \mathrm{s}$ which becomes even more pronounced at higher $r_{\mathrm{f}}$ (Figure 4). This suggests that there is a dynamic change in the force-dependent unbinding pathways from states $\mathrm{LR}_{1}$ and $\mathrm{LR}_{2}$. Unlike the distribution of unbinding forces, $p(t)$ for all complexes increases and becomes narrow around shorter unbinding times as $r_{\mathrm{f}}$ is increased (insets in Figure $4)$. Faster decay of $p(t)$ for P-selectin complexes compared with complexes involving L-selectins also indicates weaker P-selectin bonds.

The analysis of the experimental data using our model shows that, for selectin complexes with antibodies, $y_{1}=y_{2}$ and $k_{10}=$ $k_{20}$ (see Table 1). This allows us to obtain analytical expressions for $p(t)$ and $p(f)$ for G1 and DREG56. Both parts of eq 6 can be added to yield

$$
\langle\dot{P}(t)\rangle=-k(t)\langle P(t)\rangle
$$

where $k=k_{0} \exp \left[y r_{\mathrm{f}} t / k T\right], k_{0} \equiv k_{10}=k_{20}$, and $y \equiv y_{1}=y_{2}$. Then, $p(t)$ is given by

$$
p(t) \equiv k(t)\langle P(t)\rangle=k(t) \mathrm{e}^{-\int_{0}^{t} d s k(s)}
$$

and $p(f)$ is computed by rescaling

$$
\begin{aligned}
p(f) & \equiv \frac{1}{r_{\mathrm{f}}} k(f)\left\langle P\left(\frac{f}{r_{\mathrm{f}}}\right)\right\rangle \\
& =\frac{k_{0}}{r_{\mathrm{f}}} \exp \left[\frac{f y}{k T}-\frac{k_{0} k T}{y r_{\mathrm{f}}}\left(\mathrm{e}^{y f / k T}-1\right)\right]
\end{aligned}
$$

The calculated $p(f)$ and $p(t)$ for P-selectin-G1 and L-selectinDREG56 complexes using the parameters in Table 1 are displayed in Figure 5. Because $k_{0}$ for G1 is an order of magnitude smaller than $k_{0}$ for DREG56 (Table 1), $p(f)$ and $p(t)$
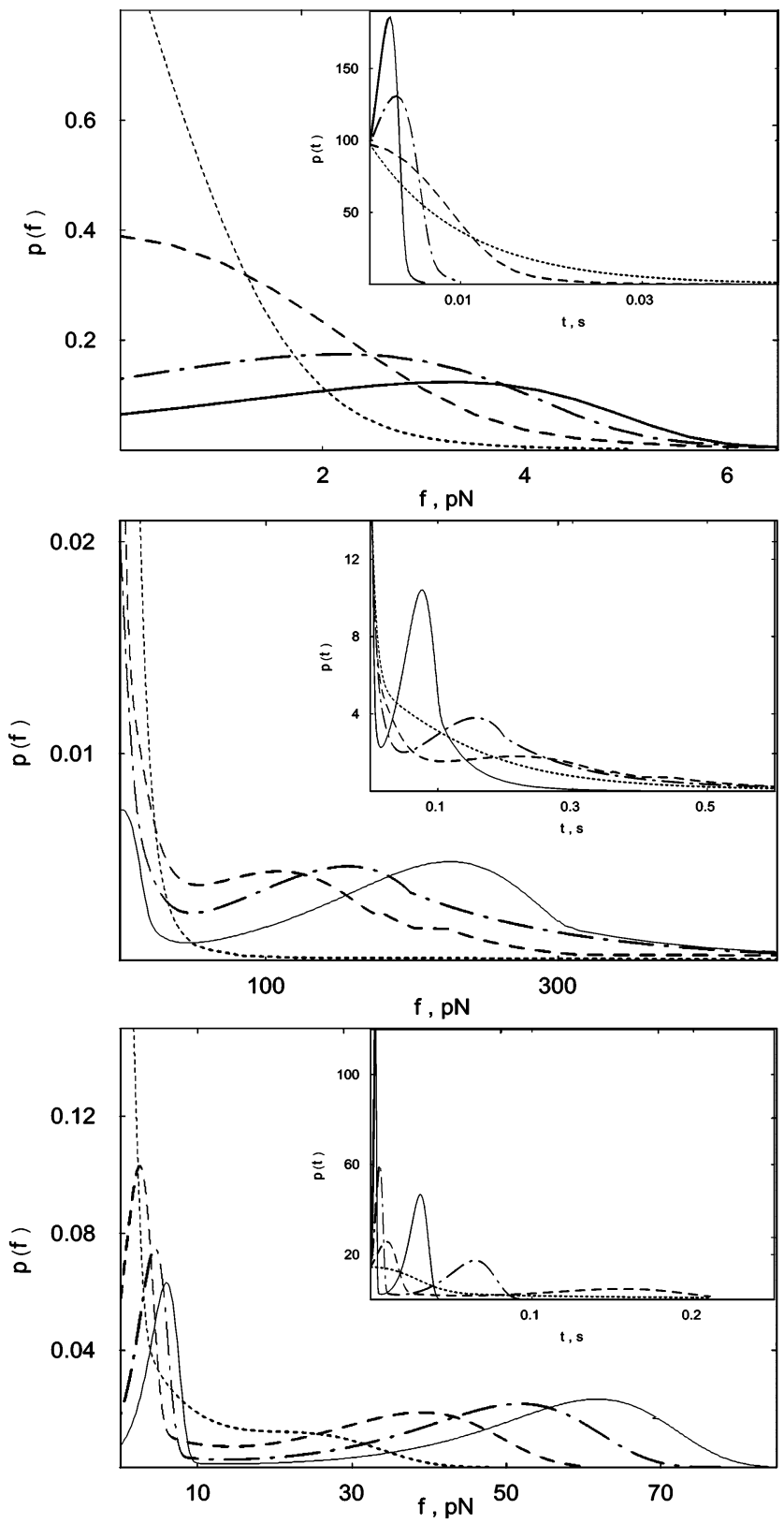

Figure 4. Distribution of unbinding forces $(p(f))$ and unbinding times $(p(t))$ (insets) for the P-selectin complex with sPSGL-1 (top), the L-selectin complex with sPSGL-1 (middle), and the L-selectinendoglycan complex (bottom). The loading rates are $r_{\mathrm{f}}=100 \mathrm{pN} / \mathrm{s}$ (dotted line), $250 \mathrm{pN} / \mathrm{s}$ (dashed line), $500 \mathrm{pN} / \mathrm{s}$ (dash-dotted line), and $1500 \mathrm{pN} / \mathrm{s}$ (solid line) for P-selectin-sPSGL-1; $r_{\mathrm{f}}=60 \mathrm{pN} / \mathrm{s}$ (dotted line), $450 \mathrm{pN} / \mathrm{s}$ (dashed line), $900 \mathrm{pN} / \mathrm{s}$ (dash-dotted line), and 2500 $\mathrm{pN} / \mathrm{s}$ (solid line) for L-selectin-sPSGl-1; $r_{\mathrm{f}}=60 \mathrm{pN} / \mathrm{s}$ (dotted line), $250 \mathrm{pN} / \mathrm{s}$ (dashed line), $800 \mathrm{pN} / \mathrm{s}$ (dash-dotted line), and $2000 \mathrm{pN} / \mathrm{s}$ (solid line) for L-selectin-endoglycan. $p(f)$ decreases in amplitude but develops a peak moving toward a larger value of $f$ as $r_{\mathrm{f}}$ is increased; in contrast to the P-selectin-sPSGL-1 complex, $p(f)$ for complexes involving L-selectins develops a second peak. For sPSGL-1 complexes with P- and L-selectins, $p(t)$ has a peak at $r_{\mathrm{f}} \approx 350 \mathrm{pN} / \mathrm{s}$; in contrast, $p(t)$ for the L-selectin-endoglycan complex develops a second peak at $r_{\mathrm{f}} \approx 250 \mathrm{pN} / \mathrm{s}$. For all three complexes, $p(t)$ approaches short unbinding times and the width decreases with increasing $r_{\mathrm{f}}$.

for G1 show slower decay with $f$ and $t$ compared with $p(f)$ and $p(t)$ for DREG56 (compare, e.g., the $p(f)$ 's and $p(t)$ 's for $r_{\mathrm{f}}=$ $500 \mathrm{pN} / \mathrm{s}$ in Figure 5). This agrees with our findings for $\langle t\rangle$ and $P(t)$ for these ligands. Just as in the case of P- and L-selectin complexes with physiological ligands, the $p(f)$ value for antibodies narrows around shorter forces (barely changing the width) 

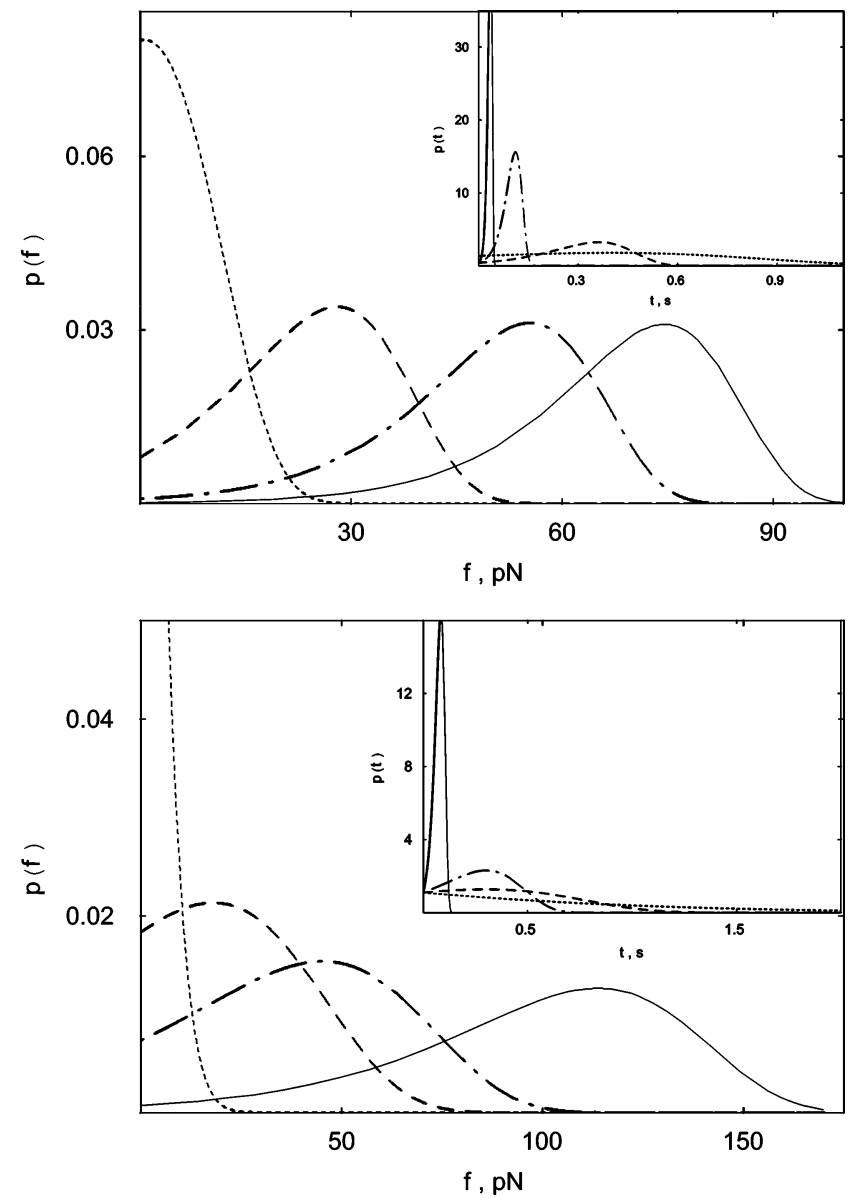

Figure 5. Distribution of unbinding forces $(p(f))$ and unbinding times $(p(t))$ (insets) for the P-selectin complex with G1 (top) and the L-selectin complex with DREG56 (bottom). The loading rates are $r_{\mathrm{f}}=20 \mathrm{pN} / \mathrm{s}$ (dotted line), $100 \mathrm{pN} / \mathrm{s}$ (dashed line), $500 \mathrm{pN} / \mathrm{s}$ (dash-dotted line), and $1500 \mathrm{pN} / \mathrm{s}$ (solid line) for the P-selectin-G1 complex and $r_{\mathrm{f}}=60 \mathrm{pN} / \mathrm{s}$ (dotted line), $150 \mathrm{pN} / \mathrm{s}$ (dashed line), $500 \mathrm{pN} / \mathrm{s}$ (dash-dotted line), and $1500 \mathrm{pN} / \mathrm{s}$ (solid line) for the L-selectin-DREG56 complex. In contrast to the P- and L-selectin complexes with sPSGL-1 and endoglycan, $p(t)$ and $p(f)$ for the G1 and DREG56 complexes with selectins have only one peak.

and $p(t)$ shifts toward longer times at faster $r_{\mathrm{f}}$. This implies that, in contrast to unbinding times, increasing $r_{\mathrm{f}}$ leads to unbinding occurring at larger forces. ${ }^{3,19}$ Both $p(f)$ and $p(t)$ for antibodies G1 and DREG56 remain single-peaked which indicates that there is only a single bound state, LR.

Let us now compare $p(f)$ and $p(t)$ obtained for physiological ligands with those obtained for antibodies. Because G1 and DREG56 possess a higher affinity to, respectively, P- and L-selectins (compare k's and y's in Table 1), $p(t)$ for $\mathrm{G} 1$ and DREG56 exhibits an order of magnitude slower decay in time as compared with $p(t)$ for sPSGL-1 and endoglycan. For a given $r_{\mathrm{f}}$ value, $p(t)$ for G1 and DREG56 has a peak that is somewhat smeared out (especially for DREG56) at slower $r_{\mathrm{f}}$. In contrast, $p(t)$ for sPSGL-1 and endoglycan shows a peak only at $r_{\mathrm{f}}>$ $300 \mathrm{pN} / \mathrm{s}$ (top panels in Figures 4 and 5). The peak position of $p(t)$, which approaches zero with decreasing width, implies higher unbinding at faster $r_{\mathrm{f}}$ for physiological ligands as well as antibodies. In contrast to $p(t=0), p(f=0)$ decreases as $r_{\mathrm{f}}$ increases for all complexes. Comparison of $p(f)$ and $p(t)$ for antibodies and physiological ligands at a fixed $r_{\mathrm{f}}$ value shows that, although selectin receptors form tighter adhesion complexes with G1 and DREG56, a linear increase in the applied force affects the stability of complexes with antibodies more profoundly compared to complexes with specific ligands, especially complexes involving P-selectins. The presence of the forcestabilized bound state $\mathrm{LR}_{2}$ for sPSGL-1 and endoglycan facilitates a dynamical mechanism for alleviating the applied mechanical stress with higher efficiency, compared with singlestate Michaelis-Menten kinetics, $\mathrm{L}+\mathrm{R} \leftrightharpoons \mathrm{LR}$ for $\mathrm{G} 1$ and DREG56. For $r_{\mathrm{f}}$ values ranging between 1000 and $1500 \mathrm{pN} / \mathrm{s}$, $p(f)$ for the P-selectin-G1 complex spans a wider range of unbinding forces $(0-120 \mathrm{pN})$, whereas the force variation is reduced to $0-10 \mathrm{pN}$ for the P-selectin complex with sPSGL-1 in the same range of $r_{\mathrm{f}}$.

Recently, Evans et al. have reported $P(f)$ for unbinding of sPSGL-1 from P-selectin. Our results at low forces (see top panel in Figure 4) qualitatively agree with the experimental results. ${ }^{19}$ Because the range of $f$ explored in ref 19 lies outside the catch-slip transition regime at constant $f$ (ref 19), a detailed comparison cannot be made. In addition, the energy landscape parameters vary dramatically as $r_{\mathrm{f}}$ is changed. ${ }^{32}$ Hence, the landscapes explored at low constant $f$ and varying $r_{\mathrm{f}}$ are markedly distinct. As a result, quantitative agreement at high $r_{\mathrm{f}}$ values using parameters from low constant $f$ values cannot be expected.

\section{Concluding Remarks}

We have extended the BT two-state mode ${ }^{20}$ previously used to analyze the catch-slip transitions in constant force unbinding of ligands from P-selectin to forced rupture of L-selectin-ligand complexes. The crux of the two-state model is that the selectins interacting with ligands can exist in two distinct bound states, $\mathrm{LR}_{1}$ and $\mathrm{LR}_{2}$. The dual catch-slip character emerges from the kinetics of the two-state model which allows for a shift in the force-induced equilibration, $\mathrm{LR}_{1} \rightleftharpoons \mathrm{LR}_{2}$. With four parameters (see Figure 1) that globally characterize the energy landscape of the cell adhesion complex, the dependence of the lifetime on the external force can be completely described. In particular, the model fully captures the catch-slip bond transitions for sPSGL-1 and naturally reveals that unbinding of the antibody has only slip bond character.

Here, we have used our theory to construct the energy landscapes of L-selectin complexes with physiological ligands, such as sPSGL-1 and endoglycan, and the antibody DREG56 using the experimental data. ${ }^{17}$ The computed average bond lifetimes $(\langle t\rangle)$ for L-selectin complexes with these ligands were compared with similar quantities for previously studied Pselectin complexes with sPSGL-1 and the antibody G1. Because forced stretching of P- and L-selectin complexes with the physiological ligands sPSGL-1 and endoglycan couples conformational relaxation and unbinding, $\langle t\rangle$ for specific proteinprotein complexes grows at low force, followed by a crossover to a decay to zero at higher force. Such a transition from the catch regime to the slip regime of unbinding suggests that the biphasic response of adhesion complexes with physiological ligands under tension is a distinctive property of the selectin family of receptors. In contrast, $\langle t\rangle$ for P- and L-selectin complexes with G1 and DREG56 decay monotonically as $f$ increases over the entire force range. We infer that selectins form stronger adhesive bonds with antibodies, compared to specific ligands. Furthermore, unbinding of selectins bound to antibodies occurs from a single bound state.

We used our model and estimated parameters to obtain testable experimental predictions for the distributions of unbinding times $(p(t))$ and unbinding forces $(p(f))$ at finite pulling speeds. These quantities can be measured by varying the loading rate. Populations of bound receptor states $(P(t))$ for selectin complexes with sPSGL-1 and endoglycan rapidly decay in $t$ at 

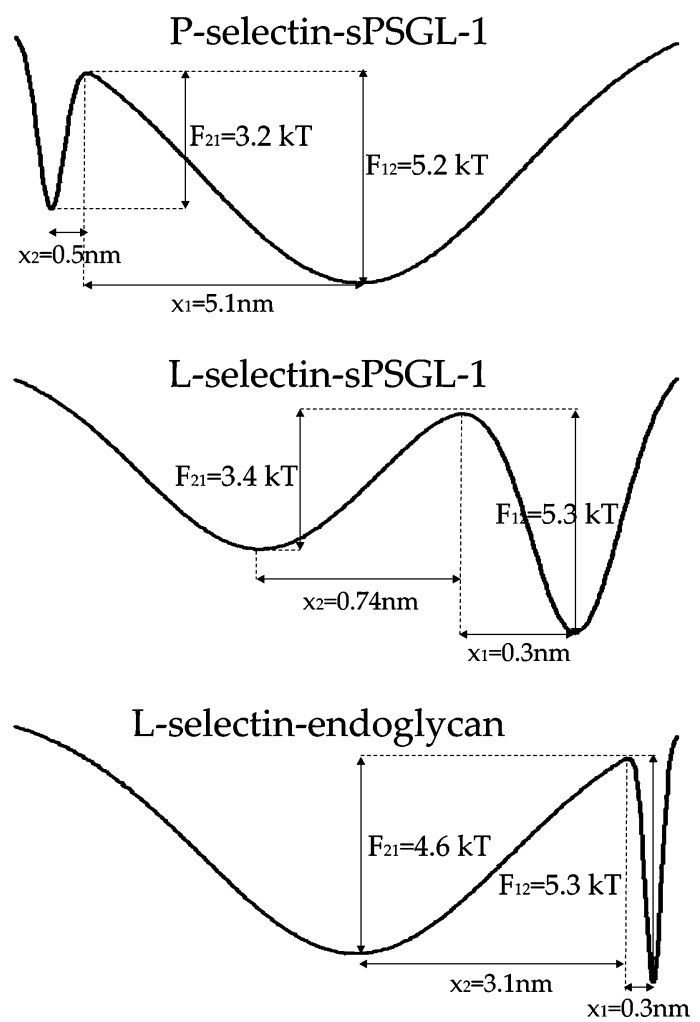

Figure 6. Schematic of the conformational free-energy profiles for the P- and L-selectin complexes with physiological ligands sPSGL-1 and endoglycan. The free-energy barriers for the conformational transitions $\mathrm{LR}_{1} \rightarrow \mathrm{LR}_{2}$ and $\mathrm{LR}_{2} \rightarrow \mathrm{LR}_{1}$ are $F_{12}$ and $F_{21}$, respectively, while $x_{1}$ and $x_{2}$ are the distances from the minima of states $\mathrm{LR}_{1}$ and $\mathrm{LR}_{2}$ to the transition state.

forces below and above the critical force $\left(f_{\mathrm{c}}\right)$, indicating a fast unbinding rate $\left(k_{\text {eff }}\right)$. However, decay of $P(t)$ is retarded for $f \approx$ $f_{\mathrm{c}}$ and slows down for $f<f_{\mathrm{c}}$ due to the decreasing rate for catch bonds, $k_{\text {eff }}=k_{\text {catch. }}$ After reaching a maximum at $f=f_{\mathrm{c}}, P(t)$ decays faster when $f>f_{\mathrm{c}}$ due to the increasing rate for slip bonds, $k_{\text {eff }}=k_{\text {slip. }}$. The crossover from a decrease at low force to an increase at high force unbinding rate results in the transition from catch to slip bonds observed for selectin complexes with physiological ligands. In contrast, the population of a single bound state of selectins complexed with G1 and DREG56 shows faster decay in time at higher force.

The force-free rates $r_{10}$ and $r_{20}$ for conformational transitions $\mathrm{LR}_{1} \rightarrow \mathrm{LR}_{2}$ and $\mathrm{LR}_{2} \rightarrow \mathrm{LR}_{1}$ enable us to estimate the freeenergy difference $(\Delta F)$ between bound selectin states $\mathrm{LR}_{1}$ and $\mathrm{LR}_{2}$, and the corresponding free-energy barriers $F_{12}$ and $F_{21}=$ $\Delta F-F_{12}$ for sPSGL-1 and endoglycan. We obtain $\Delta F$ by equating $r_{12}$ and $r_{21}$ which leads to $\Delta F=k T \ln \left[r_{20} / r_{10}\right]$. We found that $\Delta F=2 k T$ and $1.9 k T$ for $\mathrm{P}$ - and L-selectin complexes with sPSGL-1 and only $0.7 k T$ for the L-selectin-endoglycan complex. From the assumption that when $P_{1} \ll P_{2}$ the freeenergy barrier for transition $\mathrm{LR}_{1} \rightarrow \mathrm{LR}_{2}$ vanishes, we found a similar barrier height: $F_{12}=5.2 \mathrm{kT}, 5.3 \mathrm{kT}$, and $5.3 \mathrm{kT}$ for the P-selectin-sPSGL-1, L-selectin-sPSGL-1, and L-selectinendoglycan complexes, respectively. Because of this assumption, the estimated values of the free-energy barriers should be considered as lower bounds. However, for complexes that are stabilizied by noncovalent interactions, this assumption is likely to be valid.

The conformational free-energy profiles for P- and L-selectin complexes with the physiological ligands sPSGL-1 and endoglycan are presented in Figure 6. Our calculations indicate that although P- and L-selectins have high sequence similarity, ${ }^{33}$ the underlying shapes of the energy landscape for $\mathrm{P}$ - and L-selectin complexes with their ligands are markedly different. Selectin complexes with sPSGL- 1 have similar $F_{12} \approx 5 k T, F_{21}$ $\approx 3 k T$, and $\Delta F=F_{12}-F_{21} \approx 2 k T$, implying that the $\mathrm{LR}_{1}$ state is more stable at zero force. Surprisingly, the $\mathrm{LR}_{1}$ and $\mathrm{LR}_{2}$ states of the L-selectin-endoglycan complex are almost equally stable $\left(F_{12} \approx F_{21}, \Delta F<k T\right)$. Similar $\Delta F$ and $F_{12}, F_{21}$ for $\mathrm{P}-$ and L-selectin complexes with sPSGL-1 and different $\Delta F$ and $F_{12}$ for SPSGL-1 and endoglycan complexes with L-selectins suggests that the relative stability of bound receptor states is determined by the nature of the ligand. Because $x_{1}+x_{2}=$ $5.6 \mathrm{~nm}$ for P-selectin-sPSGL-1, and $\approx 3.4 \mathrm{~nm}$ for the L-selectin-endoglycan complex, and only $\approx 1 \mathrm{~nm}$ for the L-selectinsPSGL-1 complex, the bound states for P-selectins are structurally more distinct, compared to the bound states for L-selectins. States $\mathrm{LR}_{2}$ of the P-selectin-sPSGL- 1 complex $\left(x_{2}=0.5 \mathrm{~nm}\right)$ and $\mathrm{LR}_{1}$ of the L-selectin-endoglycan complex $\left(x_{1}=0.3 \mathrm{~nm}\right)$ are close to the transition state (Figure 6 ). The shorter $\approx 1 \mathrm{~nm}$ distance between the minima of $\mathrm{LR}_{1}$ and $\mathrm{LR}_{2}$ for the L-selectinsPSGL-1 complex (compared with the $5.6 \mathrm{~nm}$ distance for the sPSGL-1 complex with P-selectin) indicates a stiff binding interface which explains, in part, the inability of L-selectins to form a double bond with the dimeric ligand PSGL-1. ${ }^{17}$

Our findings indicate that the two interconverting bound states of P- or L-selectin receptors, characterized by the forcemodulated relative thermodynamic stability, provide an efficient mechanism for relieving abrupt mechanical stress by prolonging the lifetimes of their complexes with physiological ligands. The resulting biphasic response of specific cell adhesion complexes to external stress meets the requirement of the shear threshold that appears to be vital for selectin-mediated cell rolling. In the range of shear forces corresponding to a pulling force below $f_{\mathrm{c}}$, increased lifetimes of cell adhesion bonds permit transient capture of cells by selectin receptors of the vascular surfaces, resulting in momentum transfer from translational motion to their rotational motion (catch regime). Due to the decreased bond lifetime above the critical force, the rolling cell is released to find the next available selectin receptor (slip regime) to which it binds. Statistically, contributions from repeated forcemodulated transition from the catch regime to the slip regime of unbinding of many single adhesion bonds add up to make the cyclic tethering and rolling of the entire cells possible. The resulting rolling motion of leukocytes in the direction of blood flow in one dimension facilitates more efficient search for bacterial infection or tissue injury compared to purely translational Brownian motion in three dimensions.

The use of the most general two-state model (eqs 10-12) to fit the available experimental data requires a few comments. From Figure 1 and eq 10, it follows that only four parameters, $r_{12}, r_{21}, k_{1}$, and $k_{2}$, are needed to obtain $\langle t\rangle$, and the lifetime distribution function $(P(t, f))$ (Figures 2 and 3$)$. It has been erroneously asserted ${ }^{22,23}$ that our formulation requires seven parameters to obtain the global observable like the average lifetime as a function of $f$. However, merely using a kinetic model to fit experimental data does not provide insight into the energy landscape governing cell adhesion complexes. To go beyond the experimental measurements, we have used the extracted values of the parameters using the two-state model to predict the minimal parameters (seven in total) of the energy landscape including the locations of transition states and freeenergy barriers. Indeed, only by comparing these parameters (Figure 6), can one distinguish, in detail, the differences in the interactions between L- and P-selectins and the ligands. To do so involves deconvolution of the global parameters $\left(r_{12}, r_{21}, k_{1}\right.$, 
and $k_{2}$ ) in terms of $\sigma, y_{1}$, and $y_{2}$ (Table 1 ). Previous studies ${ }^{22,23}$ have not undertaken this exercise.

The two-state model ${ }^{19,20}$ and the exact kinetic equations that we proposed in our previous study ${ }^{20}$ have been used verbatim by Thomas et al. ${ }^{26}$ to explain the shear-enhanced FimH-mediated adhesion. These authors state ${ }^{26}$ that in our earlier study we used "assumptions suited to explain the single-exponential decay selectin data". We used the experimental data and the two-state model without assuming rapid interconversion between the bound states. The resulting parameters revealed that the lifetime distribution is single-exponential for P-selectin-sPSGL-1 unbinding which is justified, as shown in ref 19 , by experiments. As shown here (eq 10) and in our previous study, ${ }^{20}$ the doubleexponential relaxation naturally follows from the kinetic analysis of the two-state model. Apparently, P-selectin-sPSGL-1 unbinding is adequately described by eq 14 , whereas the full kinetic equations (eq 10) may be necessary for describing the data for bacterial adhesion. ${ }^{26}$

Acknowledgment. This work was supported by National Science Foundation grant NSFCHE-05-14056.

\section{References and Notes}

(1) Springer, T. A. Cell 1994, 76, 301

(2) Springer, T. A. Nature 1990, 346, 425.

(3) Evans, E.; Leung, A.; Hammer, D.; Simon, S. Proc. Natl. Acad. Sci. U.S.A. 2001, 98, 3784.

(4) Sako, D.; Comess, K. M.; Barone, K. M.; Camphausen, R. T. Cummings, D. A.; Shaw, G. D. Cell 1995, 83, 323.

(5) Alon, R.; Chen, S.; Puri, K. D.; Finger, E. B.; Springer, T. A. J. Cell Biol. 1997, 138, 1169.

(6) Lawrence, M. B.; Kansas, G. S.; Kunkel, E. J.; Ley, K. J. Cell Biol. 1997, 136, 717.

(7) Finger, E. B.; Puri, K. D.; Alon, R.; Lawrence, M. B.; von Andrian, U. H.; Springer, T. A. Nature 1996, 379, 266.

(8) Alon, R.; Hammer, D. A.; Springer, T. A. Nature 1995, 374, 539

(9) Brunk, D. K.; Goetz, D. J.; Hammer, D. A. Biophys. J. 1995, 71, 2902.

(10) Bell, G. L. Science 1978, 200, 618.
(11) Dembo, M. Lectures on Mathematics in the Life Sciences. Some Mathematical Problems in Biology; AMS: Providence, RI, 1994; Chapter 1.

(12) Pierres, A.; Banoliel, A. M.; Bongrand, P.; van der Merwe, P. A. Proc. Natl. Acad. Sci. U.S.A. 1996, 93, 15114

(13) Smith, M. J.; Berg, E. L.; Lawrence, M. B. Biophys. J. 1999, 77, 3371 .

(14) Ramachandran, V.; Yago, T.; Epperson, T. K.; Kobzdej, M. M. A.; Nollert, M. U.; Cummings, R. D.; Zhu, C.; McEver, R. P. Proc. Natl. Acad. Sci. U.S.A. 2001, 98, 10166.

(15) Merkel, R.; Nassoy, P.; Leung, A.; Ritchi, K.; Evans, E. Nature 1999, 397, 50 .

(16) Marshall, B. T.; Long, M.; Piper, J. W.; Yago, T.; McEver, R. P.; Zhu, C. Nature 2003, 423, 190.

(17) Sarangapani, K. K.; Yago, T.; Klopocki, A. G.; Lawrence, M. B.; Fieger, C. B.; Rosen, S. D.; McEver, R. P.; Zhu, C. J. Biol. Chem. 2004, 279,2291

(18) Yago, T.; Wu, J.; Wey, C. D.; Klopocki, A. G.; Zhu, C.; McEver, R. P. J. Cell Biol. 2004, 166, 913 .

(19) Evans, E.; Leung, A.; Heinrich, V.; Zhu, C. Proc. Natl. Acad. Sci. U.S.A. 2004, 101, 11281

(20) Barsegov, V.; Thirumalai, D. Proc. Natl. Acad. Sci. U.S.A. 2005, 102,1835

(21) Nevo, R.; Stroh, C.; Kienberger, K.; Kaftan, D.; Brumfeld, V.; Elbaum, M.; Reich, Z.; Hinterdorfer, P. Nat. Struct. Biol. 2003, 10, 553.

(22) Pereverzev, Y. V.; Prezhdo, O. V.; Thomas, W. E.; Sokurenko, E. V. Phys. Rev. E 2006, 72, 010903(R).

(23) Pereverzev, Y. V.; Prezhdo, O. V.; Forero, M.; Sokurenko, E. V.; Thomas, W. E. Biophys. J. 2006, 89, 1446.

(24) Pereverzev, Y. V.; Prezhdo, O. V. Biophys. J. 2006, 91, L19.

(25) Liu, F.; Ou-Yang, Z.-C.; Iwamoto, M. Phys. Rev. E 2006, 73 010901.

(26) Thomas, W.; Forero, M.; Yakovenko, O.; Nilsson, L.; Vicini, P.; Sokurenko, E.; Vogel, V. Biophys. J. 2006, 90, 753

(27) McEver, R. P. Curr. Opin. Cell Biol. 2002, 14, 581.

(28) Leppänen, A.; Yago, T.; Otto, V. L.; McEver, R. P.; Cummings, R. D. J. Biol. Chem. 2003, 278, 26391.

(29) Somers, W. S.; Tang, J.; Shaw, G. D.; Camphausen, R. T. Cell 2000, 103, 467.

(30) Hummer, G.; Szabo, A. Biophys. J. 2003, 85, 5.

(31) Chen, S.; Springer, T. A. Proc. Natl. Acad. Sci. U.S.A. 2001, 98, 950.

(32) Hyeon, C.; Thirumalai, D. Proc. Natl. Acad. Sci. U.S.A. 2005, 102, 1789.

(33) Dima, R. I.; Thirumalai, D. Protein Sci. 2006, 15, 258. 